Research Article

\title{
Propofol Combined with Fentanyl Is Superior to Propofol Alone in Sedation Protocols for Painless Gastrointestinal Endoscopy
}

\author{
Jie Chang ${ }^{1}$ and Chun Yang $\mathbb{1}^{2}$ \\ ${ }^{1}$ Department of Anesthesiology, The Second Hospital of Jiaxing, China \\ ${ }^{2}$ Department of Anesthesiology, The Affiliated Hospital of Jiaxing University, China
}

Correspondence should be addressed to Chun Yang; mail17816519327@163.com

Received 10 March 2021; Revised 26 April 2021; Accepted 29 April 2021; Published 17 May 2021

Academic Editor: Songwen Tan

Copyright (C) 2021 Jie Chang and Chun Yang. This is an open access article distributed under the Creative Commons Attribution License, which permits unrestricted use, distribution, and reproduction in any medium, provided the original work is properly cited.

\begin{abstract}
Sufficient propofol or fentanyl doses necessary to prevent the response to skin incision do not necessarily reduce hemodynamic responses during surgery. The purpose of this study was to characterize the pharmacodynamic interaction between propofol and fentanyl with respect to the sedative effects and safety during painless gastrointestinal endoscopy. From October 2018 to October 2020, 200 patients undergoing painless gastrointestinal endoscopy in our department's outpatient or inpatient clinic were selected and randomly divided into a control group and an observation group, 100 patients per group. Prior to surgery, the patients in the two groups were required to be connected to an electrocardiogram monitor and then were instructed to lie on the left side and receive continuous oxygen infusion of $2-3 \mathrm{~L} / \mathrm{min}$ with a nasal cannula. The control group was injected with propofol injection $(100 \mathrm{mg} / \mathrm{min})$. The observation group was given fentanyl intravenous injection at a dose of $0.1 \mu \mathrm{g} / \mathrm{kg}$ followed by propofol injection $(100 \mathrm{mg} / \mathrm{min})$. Painless gastrointestinal endoscopy was performed after the patients entered a sleep state and the eyelash reaction disappeared. Outcome analysis was performed on preoperative and intraoperative hemodynamic indicators, including heart rate, blood oxygen saturation, dose of propofol, time for endoscopy, recovery time, hospitalization after recovery, Montreal Cognitive Assessment (MoCA) score, and Mini-Mental State Examination (MMSE) score. The incidence rate of adverse reactions in the observation group was $6 \%$, which was notably lower than that of the control group $(18 \%)$. The total response rate of the observation group was $98 \%$, which was significantly higher than that of the control group (90\%) $(P<0.05)$. The intraoperative heart rate and blood oxygen saturation of the observation group were higher than those of the control group $(P<0.0 \mathrm{ss})$. The patients receiving sedation with propofol plus fentanyl had fewer doses of propofol and shorter recovery time than those receiving propofol alone $(P<0.05)$. It was also revealed that the patients receiving sedation with propofol plus fentanyl exhibited more MoCA and MMSE scores than those receiving propofol alone 30 min after sedation during painless gastrointestinal endoscopy. Taken together, sedation with propofol combined with fentanyl was more effective and safer than that with propofol alone in painless gastrointestinal endoscopy, which can ensure a wake-up state, stable breathing cycle, and better gastrointestinal painless procedure.
\end{abstract}

\section{Background}

Gastrointestinal endoscopy plays an important role in the discovery of digestive tract diseases, especially early cancer, and has been gradually included in the scope of routine physical examination [1]. The endoscopy is performed to identify potentially premalignant lesions in the gastrointestinal tract, sample or resect the area of interest, await histologic results, and then plan a treatment and/or surveillance strategy [2]. Detection of subtle lesions and decision about whether they are clinically significant depend on the skills and experience of endoscopists. For patients undergoing gastrointestinal endoscopy, most of them still need to rely on the effect of anesthesia to achieve a stable examination, so as to reduce the patient's fear of the examination and make comfortable medical treatment more extensive and safer [3]. The 
anesthesia requirements and technical level of painless gastrointestinal endoscopy are also constantly updated and improved [4]. However, because gastrointestinal endoscopy is an intrusive operation, patients often experience various degrees of stress, fear, nausea, increased blood pressure, and pulse speed when undergoing the examination [5]. Some patients even refuse the examination because of fear, leading to delays in diagnosis and treatment.

Endoscopic diagnosis and treatment are safe, minimally invasive, and effective diagnosis and treatment methods commonly used in gastroenterology clinics. It is currently a rapidly developing, mature technology and widely used clinical medical technology. However, in the process of endoscopic diagnosis and treatment, patients usually have varying degrees of tension, fear, anxiety, nausea, bloating, abdominal pain, and other discomforts, which often affect the examination. In recent years, painless endoscopy is a good aid to gastrointestinal endoscopy through the application of anesthetics [6]. It has the characteristics of being painless and more humane, low missed diagnosis rate, and no serious complications. It is currently widely used in clinical practice. However, propofol for injection, which is routinely used in painless gastrointestinal endoscopy, has no analgesic effect. When encountering strong painful stimuli, patients still respond and affect operation [7].

How to obtain a better anesthesia effect, shorten the awakening time, and improve the comfort of patient examinations requires continuous exploration and research. Painless gastrointestinal endoscopy is a common clinical method to diagnose and treat digestive system diseases, which can minimize the suffering of patients [8]. Therefore, it is favored by patients and physicians. In the process of painless gastrointestinal endoscopy, it is often necessary to use propofol for anesthesia. It has a good anesthesia and sedative effect, and it has fast onset and can make the patient wake up quickly, but it will also affect the patient's respiratory system and heart. The blood vessels have a certain inhibitory effect, and no better analgesic effect can be achieved. In this study, we recruited 200 patients undergoing painless gastrointestinal endoscopy to characterize the pharmacodynamic interaction between propofol and fentanyl with respect to the sedative effects and safety during painless gastrointestinal endoscopy.

\section{Materials and Methods}

2.1. Ethical Statement. The MoCA and MMSE scales were performed with the approval of the Ethics Committee of the Affiliated Hospital of Jiaxing University.

2.2. Inclusion and Exclusion Criteria. The study subjects in this study should meet the following inclusion and exclusion criteria. Inclusion criteria were as follows: (1) requirement for gastrointestinal endoscopy; (2) I-II grades according to the American Society of Anesthesiologists (ASA) Classification System which was developed to offer clinicians a simple categorization of a patient's physiological status that can be helpful in predicting operative risk [9]; (3) education level reaching primary school or above; (4) age ranging from 18 to 70 years old; and (5) providing informed content. Exclusion criteria were as follows: (1) those with contraindications to endoscopy; (2) those allergic to the medication used in this observation; (3) those with severe obstructive pulmonary disease and severe snoring; (4) those with cognitive impairments or neurological disorders; and (5) those with severe heart, liver, and kidney dysfunction.

2.3. Participants. From October 2018 to October 2020, 200 patients who underwent painless gastrointestinal endoscopy in our department's outpatient or inpatient clinic were selected as study subjects who meet the inclusion and exclusion criteria. These 200 patients were randomly arranged into the control group and observation group, 100 per group. The control group consisted of 48 males and 52 females, aged 22 to 70 years old, with an average age of $42.46 \pm 5.63$ years and an average weight of $66.5 \pm 8.2 \mathrm{~kg}$. The patients in the control group underwent painless gastrointestinal endoscopy for the following reasons: chronic inflammation of the digestive tract $(n=30)$, physical examination $(n=27)$, esophageal reflux $(n=12)$, gastric polyps $(n=9)$, intestinal polyps $(n=10)$, constipation $(n=9)$, and lower abdominal pain $(n=3)$; gastroscopy was performed in 54 cases and colonoscopy in 46 cases. The observation group consisted of 45 males and 55 females, aged 22 to 71 years old, with an average age of $46.22 \pm 5.19$ years and an average weight of $68.3 \pm 7.5 \mathrm{~kg}$. The patients in the observation group underwent painless gastrointestinal endoscopy for the following reasons: physical examination $(n=28)$, chronic inflammation of the digestive tract $(n=26)$, gastric polyps $(n=12)$, gastric ulcer $(n=12)$, intestinal polyps $(n=12)$, constipation $(n=6)$, and lower abdominal pain $(n=4)$; gastroscopy was performed in 56 cases and colonoscopy in 44 cases. The two groups exhibited no significant differences in sex, age, weight, and disease $(P>0.05)$, and they were comparable.

2.4. Sedation Protocols. Eight hours before painless gastrointestinal endoscopy, the patients in the two groups were required to abstain from food and drink, be given an indwelling needle, have the venous channel open by intravenous infusion of normal saline, and be connected to the ECG monitor to observe and record the patient's blood pressure, heart rate, and oxygen saturation After confirming that they are within the normal range, the patient is instructed to lie on the left side and given continuous oxygen infusion of 2 $3 \mathrm{~L} / \mathrm{min}$ with a nasal cannula. The control group was injected slowly with propofol injection (Guangdong Jiabo Pharmaceutical Co., Ltd., National Medicine Zhunzi H20051842), and the injection speed was $100 \mathrm{mg} / \mathrm{min}$; the observation group was given fentanyl intravenously at a dose of $0.1 \mu \mathrm{g} / \mathrm{kg}$ and then injected with propofol, and the injection speed is $100 \mathrm{mg} / \mathrm{min}$. Painless gastrointestinal endoscopy can be performed after the patient enters a sleep state and the eyelash reaction disappears. Both groups used our Olympus 260 series electronic gastrointestinal endoscopy for inspection operations. Gastroscopy can usually be done with a single dose. Propofol can be added to colonoscopy according to the situation. During the examination, blood pressure, heart rate, oxygen saturation, and patient response are closely 
monitored, and the dosage of propofol and the patients's wake-up time after anesthesia are recorded. Those with abnormal blood pressure and heart rate can be treated symptomatically. Record the patient's blood pressure, heart rate, and blood oxygen saturation before and after the examination.

2.5. Evaluation of Patient Outcomes. The sedative effects of anesthetic drugs were evaluated as follows: 80-100 points indicate excellent response (namely, the patient has no adverse reactions and the painless gastrointestinal surgery is successfully finished); 80-100 points indicate good response (namely, the patient experiences mild adverse reactions and the painless gastrointestinal surgery is basically finished); and less than 60 points indicate poor response (namely, the patient experiences serious adverse reactions and the painless gastrointestinal surgery is difficult to finish). Total response rate $=($ excellent + good response $) /$ total number of cases $\times$ $100 \%$. Adverse reactions following painless gastrointestinal surgery include intraoperative respiratory depression, vomiting, and dizziness. The hemodynamic parameters of painless gastrointestinal surgery include heart rate and blood oxygen saturation.

2.6. Mini-Mental State Examination (MMSE) Scale. In clinical and research settings, the MMSE is the most widely used instrument for the screening of cognitive impairment worldwide, which correlates well with more time-consuming Intelligence Quotient (IQ) tests, but it will not easily pick up cognitive problems caused by focal brain lesions. Scale items involve orientation (What is the time, date, day, month, and year? What is the name of this ward, hospital, district, town, and country?), registration (name three objects only once; repeat the objects until the patient can repeat them accurately), attention and calculation (ask the patient to subtract 7 from 100 and then 7 from the result four more times), recall (ask the patient to repeat the names of the three objects learnt in the registration test), and language (each of two simple objects named; an accurate repetition of the phrase; give a 3-stage command; write "Close your eyes" on a blank piece of paper and ask the patient to follow the written command; ask the patient to write a sentence. Score 1 point if the sentence is sensible and contains a noun and a verb; draw a pair of intersecting pentagons with each side approximately 1 inch long). Total maximum scores are 30 points. A score of 17 or less for illiteracy, 20 or less for primary school, and 24 or less for senior high school and above will pick up about $90 \%$ of patients with cognitive impairments, with about $10 \%$ false positives. If the postoperative score is 2 points lower than the preoperative score, it is considered that the cognitive function of patients was decreased.

2.7. Montreal Cognitive Assessment (MoCA) Scale. The MoCA is a 30-point scale containing the following subitems: visuospatial/executive functions, naming, memory, attention, language-sentence repetition, and language-verbal fluency.

Visuospatial/executive functions: shapes were changed from circle to triangle, and Roman alphabets were replaced in the trail making test part B as there was no substitute for
Roman alphabets in Chinese. The number of steps required for the completion of the task was retained.

Naming: the picture of a rhinoceros was replaced with an elephant to reflect local familiarity.

Memory: the word "velvet" was replaced with "silk" and "daisy" was replaced with "rose" to reflect local familiarity.

Attention: Arabic numerals were used instead of Roman alphabets for the reasons given above; the numbers and positions of responses were retained

Language-sentence repetition: the name used in the Chinese version was changed to a more common Chinese name to reflect local familiarity.

Language-verbal fluency: phonemic letter fluency was replaced by animal fluency as there is no letter-equivalent linguistic unit in Chinese.

A score of 26 or more indicates normal cognitive function.

2.8. Statistical Methods. The data was processed by using SPSS 21.0 statistical software. The measurement data is expressed as mean \pm standard deviation, and the $t$-test is performed. The count data is expressed as cases (percentage), and the chi-square test is performed. The difference was statistically significant $(P<0.05)$.

\section{Results}

3.1. Sedation with Propofol plus Fentanyl Inhibited the Incidence of Intraoperative Respiratory Depression during Painless Gastrointestinal Endoscopy. The incidence of adverse reactions was analyzed in sedation protocols of propofol with or without fentanyl for painless gastrointestinal endoscopy. The patients experienced adverse reactions including intraoperative respiratory depression, vomiting, and dizziness. There were 5 cases of intraoperative respiratory depression, 5 cases of vomiting, and 5 cases of dizziness in the observation group. The control group had 13 cases of intraoperative respiratory depression, 6 cases of vomiting, and 6 cases of dizziness. The two groups showed significant difference in the incidence of intraoperative respiratory depression (Figure 1, $P=0.0485$ ). However, no significant difference was detected in the total incidence of adverse reactions $(P=0.077)$.

\subsection{Propofol plus Fentanyl Conferred Better Sedative Effects} than Propofol Alone for Painless Gastrointestinal Endoscopy. In order to compare the sedative effects of protocols of propofol with or without fentanyl during painless gastrointestinal endoscopy, each patient was evaluated as having excellent response, good response, and poor response. The observation group had 88 patients with excellent response, 10 with good response, and 2 with poor response. The control group had 80 patients with excellent response, 10 with good response, and 10 with poor response. The total response rate of the observation group was $98 \%$, which was significantly higher than that of the control group (90\%) $(Z=2.382, P<0.05$, Table 1$)$.

3.3. Sedation with Propofol plus Fentanyl Maintained Hemodynamic Status during Painless Gastrointestinal 


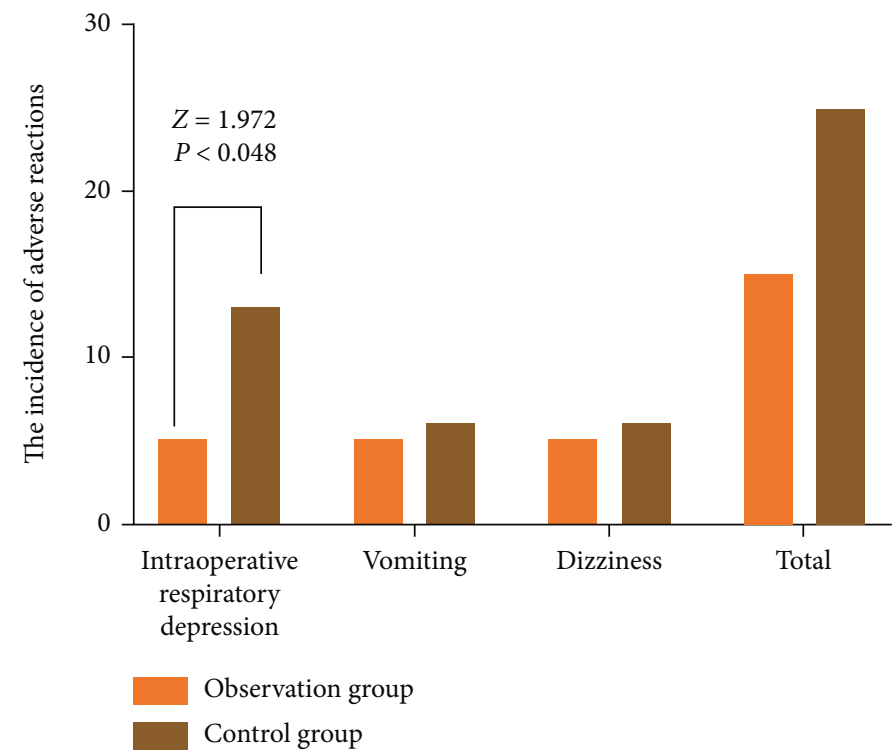

Figure 1: The incidence of adverse reactions after painless gastrointestinal endoscopy in the observation and control groups.

TABLE 1: Sedative effects of propofol plus fentanyl or propofol alone during painless gastrointestinal endoscopy.

\begin{tabular}{lcccc}
\hline & Excellent response & Good response & Poor response & Total response rate \\
\hline Observation group $(n=100)$ & 88 & 10 & 2 & 98 \\
Control group $(n=100)$ & 80 & 10 & - & 90 \\
$Z$ & & - & - & 2.382 \\
$P$ & & - & 0.017 \\
\hline
\end{tabular}

Endoscopy. Subsequent analysis was performed to examine the influence of protocols of propofol with or without fentanyl on the hemodynamic status of the patients undergoing painless gastrointestinal endoscopy. The heart rate and blood oxygen saturation were evaluated to reflect patient hemodynamic status. There was no significant difference in the heart rate and blood oxygen saturation between the two groups before painless gastrointestinal endoscopy. During the gastrointestinal endoscopy, the heart rate and blood oxygen saturation in the observation group were higher than those in the control group $(P<0.05$, Table 2$)$.

\subsection{Sedation with Propofol plus Fentanyl Promoted Patient} Recovery after Painless Gastrointestinal Endoscopy. Each patient was evaluated for dose of propofol, time for endoscopy, recovery time, and hospitalization after recovery after painless gastrointestinal endoscopy. As shown in Table 3, the patients receiving sedation with propofol plus fentanyl had fewer doses of propofol and shorter recovery time than those receiving propofol alone $(P<0.05)$, indicating that sedation with propofol plus fentanyl promoted patient recovery after painless gastrointestinal endoscopy. However, no remarkable difference was found with regard to time for endoscopy and hospitalization after recovery $(P>0.05)$.

3.5. MoCA and MMSE Scores of Patients Receiving Sedation with Propofol plus Fentanyl or Propofol Alone during Painless Gastrointestinal Endoscopy. The potential for indi- viduals to develop cognitive impairment as a consequence of major surgery with anesthesia has been increasingly recognized. We used MoCA and MMSE scales to reflect the cognitive function of patients receiving sedation with propofol plus fentanyl or propofol alone before and $30 \mathrm{~min}$ and $60 \mathrm{~min}$ after sedation during painless gastrointestinal endoscopy. The MoCA and MMSE scores between the observation group and the control group before and $60 \mathrm{~min}$ after gastrointestinal endoscopy were not statistically different $(P>0.05$ ). The patients receiving sedation with propofol plus fentanyl exhibited more MoCA and MMSE scores than those receiving propofol alone $30 \mathrm{~min}$ after sedation during painless gastrointestinal endoscopy $(P<0.05$, Figure 2$)$.

\section{Discussion}

Gastrointestinal diseases are one of the more common diseases in clinical medicine. With the continuous progress and development of social economy and culture, people's material lifestyles are changing day by day, and the incidence of gastrointestinal diseases has increased significantly. At present, the main treatment of gastrointestinal diseases in clinical medicine is painless gastrointestinal endoscopy [10]. Through gastrointestinal endoscopy, the patient's specific gastrointestinal disease can be clarified and the patient can be treated. Propofol is the commonly used anesthetic before painless gastrointestinal surgery [11]. Due to many factors, its anesthesia effect is not very obvious, and it is easy 
TABLE 2: Preoperative and intraoperative heart rate and blood oxygen saturation of patients receiving sedation with propofol plus fentanyl or propofol alone during painless gastrointestinal endoscopy.

\begin{tabular}{|c|c|c|c|c|}
\hline & \multicolumn{2}{|c|}{ Heart rate (times/min) } & \multicolumn{2}{|c|}{ Blood oxygen saturation (\%) } \\
\hline & Preoperative & Intraoperative & Preoperative & Intraoperative \\
\hline Observation group $(n=100)$ & $72.36 \pm 1.31$ & $67.98 \pm 1.22$ & $98.65 \pm 1.75$ & $96.12 \pm 1.26$ \\
\hline Control group $(n=100)$ & $72.56 \pm 1.37$ & $63.26 \pm 1.25$ & $98.36 \pm 1.69$ & $94.26 \pm 1.36$ \\
\hline$t$ & 1.055 & 27.023 & 1.192 & 10.031 \\
\hline$P$ & 0.293 & 0.0001 & 0.235 & 0.0001 \\
\hline
\end{tabular}

TABLE 3: Dose of propofol, time for endoscopy, recovery time, and hospitalization after recovery of patients receiving sedation with propofol plus fentanyl or propofol alone during painless gastrointestinal endoscopy.

\begin{tabular}{lcccc}
\hline Group & Dose of propofol $(\mathrm{mg})$ & $\begin{array}{c}\text { Time for endoscopy } \\
(\mathrm{min})\end{array}$ & $\begin{array}{c}\text { Recovery time } \\
(\mathrm{min})\end{array}$ & $\begin{array}{c}\text { Hospitalization after recovery } \\
(\mathrm{min})\end{array}$ \\
\hline Observation group $(n=100$ & $114.89 \pm 7.34$ & $15.01 \pm 6.47$ & $5.67 \pm 1.48$ & $20.97 \pm 5.32$ \\
) & $129.47 \pm 8.14$ & $16.22 \pm 7.18$ & $7.73 \pm 1.56$ & $22.17 \pm 7.49$ \\
Control group $(n=100)$ & 13.300 & 1.252 & 9.580 & 1.306 \\
$t$ & $<0.0001$ & 0.212 & $<0.0001$ & 0.193 \\
$P$ & & & \\
\hline
\end{tabular}
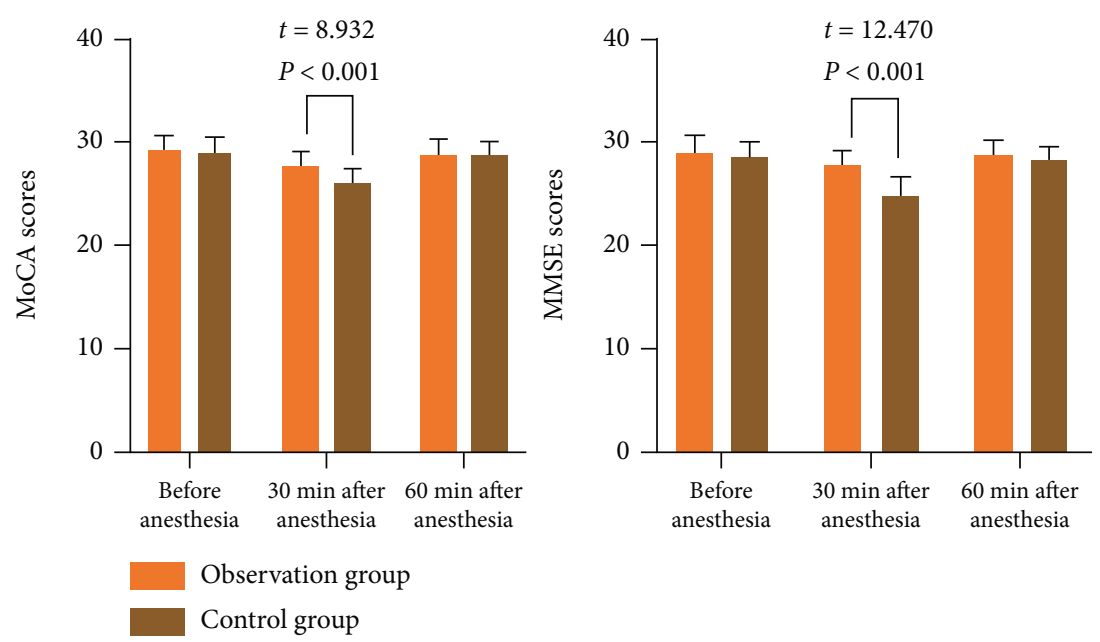

FIgURE 2: MoCA and MMSE scores of patients receiving sedation with propofol plus fentanyl or propofol alone during painless gastrointestinal endoscopy.

to cause adverse reactions in patients [12]. Many scholars in the medical field believe that propofol combined with lowdose fentanyl can not only have a good anesthesia effect but also reduce the incidence of adverse reactions in patients [13]. Therefore, in order to clarify the effect of the combination of propofol and fentanyl on painless gastrointestinal surgery, this study launched a study on the application of propofol and fentanyl. The results of this study showed that the two groups showed significant difference in the incidence of intraoperative respiratory depression. However, no significant difference was detected in total incidence of adverse reactions. In terms of response rate and preoperative and intraoperative heart rate and blood oxygen saturation and intraoperative hemodynamic comparison, the observation group was better than the control group. The reason is that the propofol drug in the control group is an anesthesiainducing drug, and its chemical name is diisopropylphenol. By activating the GABA receptor-chloride ion complex, it can inhibit the central nervous system and finally achieve sedation and hypnosis [14]. Based on the propofol drug, the observation group implemented fentanyl citrate. This drug is an analgesic with a molecular structure similar to morphine. It is combined with propofol as an anesthetic. Fentanyl is a synthetically powerful horse mouth analgesic. It is mainly absorbed through the gastrointestinal tract and fully binds to the human body proteins. It not only has a small impact on the cardiovascular function of patients but also can achieve analgesia [15]. The dosage of propofol combined with fentanyl was significantly reduced, and the patient's consciousness recovered faster after the operation was 
extubated, and the eye opening time was shorter. However, with propofol alone, the dosage is large, the breathing is easily inhibited, the recovery of consciousness after extubation after the operation is slow, and the recovery from opening eyes is slow. It can be seen that the effect of propofol combined with small dose of fentanyl is better [16].

Although gastrointestinal endoscopy does not take a long time, it can also cause a certain degree of pain to the patient during the examination. Therefore, anesthesia is usually required. The ideal anesthesia state is to ensure the stability of the patient's hemodynamic changes during the detection process; at the same time, it can be quickly induced by anesthesia, and the patient can be quickly awakened after the operation [17]. At present, propofol is usually used in gastrointestinal endoscopy. Although it has good anesthesia and sedation, it can induce anesthesia quickly and can make the patient wake up quickly after surgery, but it also affects patients. The respiratory system and cardiovascular system have inhibitory effects [18], as shown by our results. Fentanyl is a synthetic opioid receptor agonist [19]. Compared with propofol, it has a shorter induction time for anesthesia and does not have much impact on the cardiovascular system. Remifentanil is an opioid receptor agonist with a short halflife and will not affect liver and kidney function. The results of this study confirm that the application of a small amount of remifentanil combined with a small dose of propofol in a painless gastrointestinal endoscopy can achieve a very ideal anesthesia effect and can ensure that the patient is in a wakeful state and is stable. The breathing cycle of the painless gastrointestinal endoscopy is significantly improved, and its anesthesia effect is far superior to that of the conventional dose of fentanyl combined with propofol anesthesia.

In this study, the two groups showed significant difference in the incidence of intraoperative respiratory depression. However, no significant difference was detected in the total incidence of adverse reactions. The total response rate of the observation group was $98 \%$, which was significantly higher than that of the control group (90\%), and the difference was statistically significant. There was no significant difference in heart rate, blood oxygen saturation, and hemodynamics between the two groups before operation. The intraoperative heart rate and blood oxygen saturation of the observation group were higher than those of the control group, and the difference was statistically significant. The MoCA and MMSE scores of the two groups before and after anesthesia were not statistically different. The MoCA and MMSE scores of the observation group were higher than those of the control group at 30 minutes after anesthesia, and there was a statistical difference.

In summary, propofol plus fentanyl in painless gastrointestinal endoscopy can achieve a very ideal sedative effect, ensure that the patient is in a wakeful state, and ensure the patient's breathing cycle is stable, so that the safety of gastrointestinal endoscopy has been significantly improved. Propofol combined with low-dose fentanyl is safer, can reduce the incidence of adverse reactions in patients, and has certain clinical application value. Nevertheless, different doses of fentanyl combined with propofol in sedation protocols for painless gastrointestinal endoscopy will be explored in further study.

\section{Data Availability}

The data used to support the findings of this study are included within the article.

\section{Conflicts of Interest}

The authors declare that they have no conflicts of interest.

\section{References}

[1] P. Valdastri, M. Simi, and R. J. Webster 3rd, "Advanced technologies for gastrointestinal endoscopy," Annual Review of Biomedical Engineering, vol. 14, no. 1, pp. 397-429, 2012.

[2] A. M. Thaker and V. R. Muthusamy, "Advanced imaging techniques in gastrointestinal endoscopy," Journal of Laparoendoscopic \& Advanced Surgical Techniques Part A, vol. 27, no. 3, pp. 234-241, 2017.

[3] A. S. o. P. Committee, D. S. Early, J. R. Lightdale et al., "Guidelines for sedation and anesthesia in GI endoscopy," Gastrointestinal Endoscopy, vol. 87, no. 2, pp. 327-337, 2018.

[4] C. J. Rees, S. Koo, and K. W. Oppong, "Future directions in therapeutic gastrointestinal endoscopy," The Lancet Gastroenterology \& Hepatology, vol. 3, no. 10, pp. 663-664, 2018.

[5] M. R. Borgaonkar, L. Hookey, R. Hollingworth et al., "Indicators of safety compromise in gastrointestinal endoscopy," Canadian Journal of Gastroenterology, vol. 26, no. 2, pp. 7178, 2012.

[6] O. S. Lin, "Sedation for routine gastrointestinal endoscopic procedures: a review on efficacy, safety, efficiency, cost and satisfaction," Intestinal Research, vol. 15, no. 4, pp. 456-466, 2017.

[7] T. Nishizawa and H. Suzuki, "Propofol for gastrointestinal endoscopy," United European Gastroenterology Journal, vol. 6, no. 6, pp. 801-805, 2018.

[8] L. Zhang, Y. Bao, and D. Shi, "Comparing the pain of propofol via different combinations of fentanyl, sufentanil or remifentanil in gastrointestinal endoscopy," Acta Cirúrgica Brasileira, vol. 29, no. 10, pp. 675-680, 2014.

[9] D. J. Doyle, A. Goyal, P. Bansal, and E. H. Garmon, American Society of Anesthesiologists Classification, StatPearls, Treasure Island, FL, USA, 2021.

[10] J. J. McGoran, M. E. McAlindon, P. G. Iyer et al., "Miniature gastrointestinal endoscopy: now and the future," World Journal of Gastroenterology, vol. 25, no. 30, pp. 4051-4060, 2019.

[11] D. Stogiannou, A. Protopapas, A. Protopapas, and K. Tziomalos, "Is propofol the optimal sedative in gastrointestinal endoscopy?," Acta Gastroenterologica Belgica, vol. 81, no. 4, pp. 520-524, 2018.

[12] R. Zhang, Q. Lu, and Y. Wu, "The comparison of midazolam and propofol in gastrointestinal endoscopy: a systematic review and meta-analysis," Surgical Laparoscopy, Endoscopy \& Percutaneous Techniques, vol. 28, no. 3, pp. 153-158, 2018.

[13] A. Amini, A. Arhami Dolatabadi, H. Kariman et al., "Low-dose fentanyl, propofol, midazolam, ketamine and lidocaine combination vs. regular dose propofol and fentanyl combination for deep sedation induction; a randomized clinical trial," Emergency, vol. 6, no. 1, article e57, 2018. 
[14] M. Hara, Y. Kai, and Y. Ikemoto, "Propofol activates GABAA receptor-chloride ionophore complex in dissociated hippocampal pyramidal neurons of the rat," Anesthesiology, vol. 79, no. 4, pp. 781-788, 1993.

[15] F. Zhang, J. Tong, W. Qing, Z. Hu, J. Hu, and Q. Liao, “MDR1 genotypes and haplotypes are closely associated with postoperative fentanyl consumption in patients undergoing radical gastrectomy," Journal of Nanomaterials, vol. 2021, Article ID 5587399, 8 pages, 2021.

[16] H. S. Bakhamees, A. Mercan, and Y. M. El-Halafawy, "Combination effect of low dose fentanyl and propofol on emergence agitation in children following sevoflurane anesthesia," Saudi Medical Journal, vol. 30, no. 4, pp. 500-503, 2009.

[17] H. Hosseinzadeh, S. E. Golzari, E. Torabi, and M. Dehdilani, "Hemodynamic changes following anesthesia induction and LMA insertion with propofol, etomidate, and propofol + etomidate," Journal of Cardiovascular and Thoracic Research, vol. 5, no. 3, pp. 109-112, 2013.

[18] J. E. Mandel, G. R. Lichtenstein, D. C. Metz, G. G. Ginsberg, and M. L. Kochman, "A prospective, randomized, comparative trial evaluating respiratory depression during patientcontrolled versus anesthesiologist-administered propofolremifentanil sedation for elective colonoscopy," Gastrointestinal Endoscopy, vol. 72, no. 1, pp. 112-117, 2010.

[19] D. Lu, L. Jiang, C. Dai, K. Yan, and J. Gao, "Fentanyl exerts an antitumor effect on papillary thyroid cancer by regulating the miR-204/KLF5 axis," Journal of Nanomaterials, vol. 2021, Article ID 5563901, 8 pages, 2021. 